\title{
Estilo de vida e nível de atividade física de indivíduos com dor no ombro atendidos em um serviço público
}

\section{Lifestyle and physical activity level in shoulder pain individuals assisted in a public service}

\author{
Jessica Paglioto 1, Jaqueline Martins ², Cesário da Silva Souza 2, Anamaria Siriani de Oliveira
}

\section{Resumo}

Esse estudo teve como objetivo caracterizar o Perfil de Estilo de Vida Individual (PEVI) de indivíduos com dor no ombro em relação à atividade física $(\mathrm{AF})$, nutrição, controle do estresse, relacionamento social e comportamento preventivo e verificar a correlação entre o componente AF do PEVI e o domínio de incapacidade avaliado pelo Shoulder Pain and Disability Index (SPADI). Trata-se de um estudo transversal, com 100 indivíduos adultos e idosos com dor no ombro (55,3 \pm 12 anos, $83 \%$ mulheres) atendidos em um serviço público de fisioterapia em 2015/2016. Os indivíduos responderam ao questionário PEVI e ao SPADI para avaliação da dor e incapacidade do ombro. Os resultados indicam que $84 \%$ dos avaliados apresentaram PEVI de regular a ruim (pontuação $\leq 33$ ), sendo positivo (pontuação média $=2$ a 3) para comportamento preventivo e relacionamento social em $94 \%$ e $53 \%$ dos indivíduos, respectivamente. Nos demais componentes, as respostas se concentraram no perfil regular (pontuação média $=1$ a 1,99 ) e positivo, mas o componente AF apresentou o maior número de indivíduos (30\%) com perfil negativo (pontuação média < 1) e identificou que a maioria não realiza $\mathrm{AF}$ moderada a intensa e exercícios de força e alongamento muscular. Os níveis de incapacidade do ombro e de AF apresentaram uma correlação baixa (-0,179; $\mathrm{p}=0,040)$. Em conclusão, indivíduos com dor no ombro apresentam estilo de vida regular, sendo importante incentivar a prática de atividade física para melhora da qualidade de vida.

\section{Palavras-chave}

Estilo de vida; Dor musculoesquelética; Ombro.

\begin{abstract}
This study aimed to characterize the Individual Lifestyle Profile (PEVI) of individuals with shoulder pain in relation to physical activity (PA), nutrition, stress management, social relationship and preventive behavior and to verify the correlation between PA component of PEVI and the disability domain assessed by the Shoulder Pain and Disability Index (SPADI). This is a cross-sectional study with 100 adult and elderly individuals with shoulder pain (55.3 \pm 12 years, $83 \%$ female) attending in a public physiotherapy service in 2015/2016. Individuals answered the PEVI questionnaire and SPADI for shoulder pain and disability assessment. The results indicate that $84 \%$ of the patients presented PEVI from regular to poor (score $\leq 33$ ), and positive (mean score $=2$ to 3 ) for preventive behavior and social relationships in $94 \%$ and $53 \%$ of the individuals, respectively. For the other components, responses were concentrated in the regular profile (mean score $=1$ to 1.99) and positive, but the PA component had the highest number of individuals (30\%) with a negative profile (mean score <1) and identified most do not perform moderate to intense PA and muscle strength and stretching exercises. Shoulder disability levels and PA showed a low correlation $(-0.179 ; p=0.040)$. In conclusion, individuals with shoulder pain present a regular lifestyle, and it is important to encourage the practice of physical activity to improve the quality of life.
\end{abstract}

\section{Keywords}

Lifestyle, Musculoskeletal Pain; Shoulder.

\section{INTRODUÇÃO}

O estilo de vida é reconhecido como um dos fatores primordiais para a manutenção da qualidade de

1 Faculdade de Medicina de Ribeirão Preto da Universidade de São Paulo, Ribeirão Preto, SP, Brasil.

2 Faculdade de Medicina de Ribeirão Preto da Universidade de São Paulo, Departamento de Biomecânica, Medicina e Reabilitação do Aparelho Locomotor, Ribeirão Preto, SP, Brasil. vida $^{1}$ e representa um conjunto de ações cotidianas que reflete as atitudes e valores das pessoas, ou seja, a maneira como as pessoas vivem e as escolhas que fazem $^{2}$. Em relação à saúde, as escolhas se referem às atividades de lazer, hábitos alimentares, comportamentos autodeterminados ou adquiridos social ou culturalmente ${ }^{3}$. 
Atualmente, diversos países têm monitorado o estilo de vida em relação às doenças crônicas não transmissíveis (DCNT), com destaque para as doenças do aparelho circulatório, câncer, diabetes e doenças respiratórias crônicas, visto que constituem o maior problema de saúde e causa de morbimortalidade do mundo e na população brasileira ${ }^{4}$. As DCNT estão associadas, dentre outros aspectos, a comportamentos de risco relacionados ao estilo de vida para alimentação, atividade física, uso do tabaco e álcool. Em vista disso, foi elaborado pelo Ministério da Saúde brasileiro um plano de ações estratégicas para o período de 2011-2022, a fim de controlar tais condições e seus fatores de risco ${ }^{5}$. No entanto, o estilo de vida tem sido pouco investigado em condições musculoesqueléticas crônicas, que também são reconhecidas como uma das principais causas de morbidade no mundo e geram elevada porcentagem de anos de vida com incapacidade e diminuição da qualidade de vida relacionada à saúde ${ }^{6,7}$.

Estudos demonstram que fatores do estilo de vida como tabagismo, estresse e obesidade desempenham papel importante na etiologia multifatorial da dor musculoesquelética $^{8,9}$ e reconhecem a prática de atividade física de nível baixo a moderado e a obesidade como potenciais fatores de risco para a ocorrência e evolução de dor musculoesquelética ao longo do tempo ${ }^{9}$. Também têm sido observado que o tabagismo, uso de álcool e sobrepeso na adolescência predispõe à dor musculoesquelética na idade adulta ${ }^{7}$.

A dor no ombro representa uma das condições musculoesqueléticas mais comuns, com prevalência de $20 \%$ a $33 \%$ da população em geral ${ }^{10}$ e gera custos consideráveis pela incapacidade, dor e cuidados de saúde ${ }^{11}$. Além de comprometer a qualidade de vida ${ }^{12}$, as disfunções de ombro podem influenciar negativamente padrões de sono, humor e capacidade de concentração ${ }^{13}$.

Em relação aos fatores etiológicos, a dor no ombro tem sido associada ao aumento da idade, ao sexo feminino, baixo nível de escolaridade, cargas excessivas de trabalho, lesões repetitivas e a fatores do estilo de vida como tabagismo, obesidade, sedentarismo e fatores psicossociais ${ }^{14,15}$. Entretanto, ainda são poucos os estudos que analisam o estilo de vida de portadores de dor no ombro e não se sabe se tais indivíduos apresentam características semelhantes ao estilo de vida de indivíduos com doenças crônicas não transmissíveis, o que poderia subsidiar futuras ações dos programas de promoção da saúde.

Os profissionais envolvidos com a atenção primária podem contribuir para detecção precoce de fatores de risco relacionados ao estilo de vida, promovendo estratégias para o desenvolvimento de hábitos de vida saudáveis em relação à atividade física, alimentação e uso do tabaco e do álcool. Tais condutas na área da saúde, também podem incentivar a corresponsabilização do indivíduo pela própria saúde e saúde da comunidade no sentido de reduzir sintomas musculoesqueléticos e melhorar a qualidade de vida e a capacidade funcional ${ }^{16}$.

Dessa forma, o objetivo desse estudo foi caracterizar o perfil de estilo de vida de indivíduos com queixa de dor no ombro atendidos em um serviço público de fisioterapia de nível secundário, identificando os comportamentos relacionados à atividade física, nutrição, controle do stress, relacionamento social e comportamento preventivo. Além disso, buscou-se verificar neste indivíduo se existe correlação entre o nível de atividade física e o nível de incapacidade do ombro.

\section{Métodos}

\section{Tipo de Estudo}

Trata-se de um estudo observacional transversal descritivo. O estudo foi realizado em um Serviço de Fisioterapia de um Centro de Saúde Escola (CSE) vinculado a 
uma universidade pública, localizado no distrito oeste do município de Ribeirão Preto, São Paulo. O CSE se insere no nível secundário da atenção à saúde e possui serviços de pronto atendimento e consultas ambulatoriais, incluindo a fisioterapia.

\section{Amostra do estudo}

A amostra do estudo foi composta por indivíduos com queixas de dor no ombro em atendimento no serviço de fisioterapia do CSE - Cuiabá no período de 01 de janeiro de 2015 a 01 de janeiro de 2016. A queixa de dor no ombro foi considerada quando a pontuação no questionário Shoulder Pain and Disability Index (SPADI) ${ }^{17}$ foi igual ou maior a 30 pontos. Considerando que o SPADI não apresenta uma classificação da pontuação em níveis de severidade da disfunção, este ponto de corte foi estabelecido com base no estudo de Puga et $a{ }^{18}$ que testou as propriedades psicométricas da versão brasileira do SPADI em 98 pacientes com diferentes disfunções de ombro e verificou uma pontuação média do SPADI de 54,2 pontos (desvio padrão=24,7 pontos), o que pode sugerir que pacientes com disfunção de ombro apresentam uma pontuação igual ou superior a 29,5 pontos. Foram excluídos do estudo os indivíduos com dificuldade de compreender os questionários aplicados na avaliação ou indivíduos que compareceram apenas na avaliação. Todos os participantes assinaram o Termo de Consentimento Livre e Esclarecido aprovado pelo Comitê de Ética em pesquisa do CSE (Processo 39/2014).

\section{Procedimentos}

Inicialmente, foi realizada a coleta das variáveis descritivas como idade, sexo, diagnóstico clínico determinado pelo ortopedista que atendeu o paciente e que constava na guia de encaminhamento para o serviço de fisioterapia, presença de DCNT, tempo de sintomas de dor no ombro, escolaridade e profissão. Em seguida, o individuo respondeu ao questionário brasileiro Perfil do Estilo de Vida Individual (PEVI) ${ }^{19}$ para avaliação do estilo de vida e ao questionário SPADI para caracterizar a dor e a incapacidade com o ombro sintomático. As pontuações dos questionários representam as variáveis primárias do estudo.

O questionário PEVI é um dos instrumentos nacionais mais utilizados para avaliar o estilo de vida ${ }^{20}$, é simples de ser aplicado e se encontra testado para consistência interna, teoria de resposta ao item e para validade através da correlação do item intra e inter-domínios ${ }^{19-21}$. Apresenta cinco componentes referentes à nutrição, atividade física, controle do stress, relacionamento social e comportamento preventivo, totalizando 15 itens, ou seja, três itens por componente, pontuados de zero (ausência total de tal característica no estilo de vida) a três (completa realização do comportamento considerado). A pontuação varia de zero a 45 pontos, sendo que quanto maior a pontuação, melhor o estilo de vida do indivíduo.

O estilo de vida avaliado pela pontuação do PEVI é categorizado em perfil excelente ( 45 pontos), bom (de 44 a 34), regular (de 33 a 27), abaixo da média (26 a 18) e ruim (17 a zero). A pontuação de cada componente é interpretada como indicativo de perfil negativo (pontuação média menor que um), regular (pontuação média entre um e 1,99) e positivo (pontuação média entre dois e três) ${ }^{19}$.

O questionário SPADI representa uma das ferramentas mais recomendadas para avaliação das disfunções de ombro e a versão brasileira é confiável, válida e responsiva ${ }^{17,18}$. O questionário apresenta um domínio de dor com cinco itens e outro domínio de incapacidade, com oito itens, pontuados em uma escala de avaliação numérica de 10 pontos, de modo que zero indica sem dificuldade ou sem dor e dez indica não conseguiu fazer ou pior dor. A pontuação total e de seus 
domínios pode variar de 0 a 100, com pontuação mais elevada indicando maior comprometimento no ombro ${ }^{17,21}$.

Ao final da avaliação, o pesquisador coloriu o pentáculo com faixas representativas dos itens avaliados e quanto mais colorida a figura, mais adequado foi o estilo de vida do indivíduo ${ }^{19,20}$. Os pacientes levaram a figura para casa, pois representa uma forma mais fácil de avaliarem a situação do seu estilo de vida atual.

\section{Análise estatística}

As variáveis descritivas e a pontuação do SPADI e de seus componentes foram apresentadas em forma de média e desvio padrão ou por frequência absoluta e relativa. O estilo de vida determinado pela pontuação do PEVI e de seus componentes foi analisado através da frequência relativa de respostas em cada perfil.

A relação entre as médias das pontuações do domínio de incapacidade do SPADI e do componente de atividade física do PEVI foi verificada pela correlação parcial com controle das variáveis sexo, idade e escolaridade. Tais variáveis foram controladas, uma vez que Malta et $\mathrm{al}^{3}$ observaram, através de um estudo descritivo, associação entre a prática de atividade física no lazer e maior escolaridade, sexo masculino e idade jovem. A correlação parcial foi classificada como alta $(r \geq 0,70)$, moderada $(r \geq 0,40$ e $r<0,70)$ e baixa $(r<0,40)^{22}$ e realizada no SPSS Versão 17 para Windows (SPSS Inc, Chicago, IL).

\section{Resultados}

Dentre os 112 indivíduos com queixas de dor no ombro elegíveis, 100 indivíduos foram avaliados (89\%), sendo excluídos dois indivíduos por incapacidade de compreender os questionários e dez por incompatibilidade de horário para ser avaliado.

A Tabela 1 apresenta as características sociodemográficas e da disfunção de ombro dos indivíduos que apresentaram média de idade 55,3 \pm 12 anos. A maioria dos indivíduos foi de mulheres (83\%), apresentou nível de ensino fundamental incompleto (44\%) e desempenhou atividades do lar ou era aposentado (41\%). Os sintomas no ombro foram crônicos em $91 \%$ dos indivíduos e a disfunção mais frequente foi síndrome da dor subacromial (46\%). A média de dor avaliada pelo

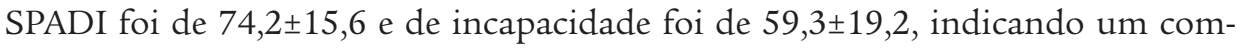
prometimento da funcionalidade do ombro de $65,0 \pm 16,3$ pontos. Em relação às DCNT, observou-se a maior ocorrência de hipertensão arterial (32\%).

Em relação à distribuição dos indivíduos por classificação da pontuação do PEVI, 84\% dos indivíduos apresentaram estilo de vida igual ou inferior a regular (37 indivíduos com perfil regular, 39 com perfil abaixo e oito com perfil ruim). O PEVI foi classificado como bom somente em 16 indivíduos e não foi classificado como perfil excelente. Os componentes Comportamento Preventivo e Relacionamento Social foram classificados como perfil positivo para maioria dos indivíduos, respectivamente, $94 \%$ e $53 \%$. Os demais componentes demonstraram uma concentração dos indivíduos no perfil regular a positivo, sendo que o domínio atividade física foi o que mais apresentou indivíduos com perfil negativo (30\%), seguido por controle do stress e nutrição (Tabela 2).

A distribuição do perfil dos indivíduos para cada item dos componentes pode ser observada na Tabela 3. O componente atividade física apresentou um maior número de respostas negativas para os itens 1 (68\%) e 2 (59\%), sendo que apenas o item 3 teve maior número de respostas positivas (65\%). O controle do stress também apresentou dois itens com maioria de respostas negativas. 
TABELA 1 - Características sociodemográficas e da disfunção dos indivíduos com dor no ombro, Ribeirão Preto, SP, $(n=100)$.

\begin{tabular}{|c|c|}
\hline Variáveis & Estimativas \\
\hline \multicolumn{2}{|l|}{ Sexo, n (\%) } \\
\hline Feminino & $83(83)$ \\
\hline Masculino & $17(17)$ \\
\hline \multicolumn{2}{|l|}{ Escolaridade, n (\%) } \\
\hline Semianalfabeto & $2(2)$ \\
\hline Ensino Fundamental & $61(61)$ \\
\hline Ensino Médio & $29(29)$ \\
\hline Ensino Superior & $8(8)$ \\
\hline \multicolumn{2}{|l|}{ Profissão, n (\%) } \\
\hline Ativo & $59(59)$ \\
\hline Aposentado ou do lar & $41(41)$ \\
\hline \multicolumn{2}{|l|}{ Tempo de sintomas, n (\%) } \\
\hline 1 a 6 meses & $9(9)$ \\
\hline$>6$ meses & $91(91)$ \\
\hline \multicolumn{2}{|l|}{ Diagnósticos, n (\%) } \\
\hline Síndrome da dor subacromial & $46(46)$ \\
\hline Lesões no ombro não especificadas & $13(13)$ \\
\hline Artrose Glenoumeral; outros & $41(41)$ \\
\hline \multicolumn{2}{|l|}{ Comorbidades, n (\%) } \\
\hline Hipertensão Arterial & $32(32)$ \\
\hline Diabetes & $15(15)$ \\
\hline Câncer & $3(3)$ \\
\hline Doenças Respiratórias Crônicas & $13(13)$ \\
\hline Idade, Média (desvio padrão) & $55,3( \pm 12)$ \\
\hline \multicolumn{2}{|l|}{ SPADI, Média (desvio Padrão) } \\
\hline Dor & $74,2(15,6)$ \\
\hline Incapacidade & $59,3(19,2)$ \\
\hline Pontuação total & $65,0(16,3)$ \\
\hline
\end{tabular}

TABELA 2 - Frequência absoluta e relativa da classificação dos componentes do PEVI em indivíduos com dor no ombro, Ribeirão Preto, SP, $(n=100)$.

\begin{tabular}{lccc}
\hline & \multicolumn{3}{c}{ Classificação, $n$ (\%) } \\
\hline Nutrição & $17(17)$ & Regular & Positivo \\
\hline Atividade Física & $30(30)$ & $37(37)$ & $46(46)$ \\
\hline Comportamento Preventivo & $0(0)$ & $39(39)$ & $31(31)$ \\
\hline Relacionamento Social & $5(5)$ & $6(6)$ & $94(94)$ \\
\hline Controle do Estresse & $19(19)$ & $42(42)$ & $53(53)$ \\
\hline
\end{tabular}

Perfil negativo: pontuação menor que 1; Perfil regular: pontuação entre 1 e 1,99; Perfil positivo: pontuação entre 2 e 3.

Verificou-se uma correlação baixa e estatisticamente significativa entre o nível de incapacidade do ombro avaliado pelo SPADI e o nível de atividade física do questionário PEVI $(-0,179 ; \mathrm{p}=0,040)$. O poder do teste a posteriori foi de 0,55. 
TABELA 3 -. Frequência de respostas positivas e negativas para os itens do questionário PEVI em indivíduos com dor no ombro, Ribeirão Preto, SP, $(n=100)$.

\begin{tabular}{|c|c|c|}
\hline & $\begin{array}{l}\text { Resposta } \\
\text { Negativa } \\
(\%)\end{array}$ & $\begin{array}{l}\text { Resposta } \\
\text { Positiva } \\
\text { (\%) }\end{array}$ \\
\hline \multicolumn{3}{|l|}{ Nutrição } \\
\hline Sua alimentação diária inclui ao menos 5 porções de frutas e verduras & 49 & 51 \\
\hline Você evita ingerir alimentos gordurosos (carnes gordas, frituras) e doces & 44 & 56 \\
\hline Você faz 4 a 5 refeições variadas ao dia, incluindo café da manhã completo & 49 & 51 \\
\hline \multicolumn{3}{|l|}{ Atividade Física } \\
\hline $\begin{array}{l}\text { Você realiza ao menos } 30 \text { minutos de atividades físicas moderadas/ intensas, } \\
\text { de forma contínua ou acumulada, } 5 \text { ou mais dias na semana }\end{array}$ & 68 & 32 \\
\hline $\begin{array}{l}\text { Ao menos duas vezes por semana você realiza exercícios que envolvam força } \\
\text { e alongamento muscular }\end{array}$ & 59 & 41 \\
\hline $\begin{array}{l}\text { No seu dia-a-dia, você caminha ou pedala como meio de transporte e, } \\
\text { preferencialmente, usa as escadas ao invés do elevador }\end{array}$ & 35 & 65 \\
\hline \multicolumn{3}{|l|}{ Comportamento Preventivo } \\
\hline $\begin{array}{l}\text { Você conhece sua PRESSÃO ARTERIAL, seus níveis de COLESTEROL e procura } \\
\text { controlá-los }\end{array}$ & 9 & 91 \\
\hline $\begin{array}{l}\text { Você NÃO FUMA e NÃO INGERE ÁLCOOL ou ingere com moderação (menos } \\
\text { de duas doses ao dia) }\end{array}$ & 19 & 81 \\
\hline $\begin{array}{l}\text { Você respeita as normas de trânsito (pedestre, ciclista ou motorista); se dirige } \\
\text { usa sempre o cinto de segurança e nunca ingere álcool }\end{array}$ & 3 & 97 \\
\hline \multicolumn{3}{|l|}{ Relacionamento social } \\
\hline Você procura cultivar amigos e está satisfeito com seus relacionamentos & 13 & 87 \\
\hline $\begin{array}{l}\text { Seu lazer inclui encontros com amigos, atividades esportivas em grupo } \\
\text { participação em associações ou entidades sociais }\end{array}$ & 37 & 63 \\
\hline $\begin{array}{l}\text { Você procura ser ativo em sua comunidade, sentindo-se útil no seu } \\
\text { ambiente social }\end{array}$ & 67 & 33 \\
\hline \multicolumn{3}{|l|}{ Controle de Estresse } \\
\hline Você reserva tempo (ao menos 5 minutos) todos os dias para relaxar & 32 & 68 \\
\hline Você mantém uma discussão sem alterar-se, mesmo quando contrariado & 56 & 44 \\
\hline Você equilibra o tempo dedicado ao trabalho com o tempo dedicado ao lazer & 52 & 48 \\
\hline
\end{tabular}

\section{Discussão}

Os resultados desse estudo demonstraram que indivíduos com dor no ombro, atendidos em um serviço de fisioterapia de nível secundário, apresentam um estilo de vida que varia de regular a ruim, sendo observado que o componente atividade física foi o que mais contribuiu para este estilo de vida desfavorável, seguido por controle de stress e nutrição. Além disso, verificou-se uma correlação baixa entre a incapacidade do ombro e o estilo de vida adotado em relação à prática de atividade física.

Os indivíduos com dor no ombro apresentaram um perfil positivo para o comportamento preventivo, com apenas 19\% dos indivíduos oferecendo respostas negativas quanto à ingestão de bebidas alcoólicas e uso de tabaco. Nossos resultados foram semelhantes ao estudo da Pesquisa Nacional de Saúde (PNS) que investigou o estilo de vida da população brasileira ${ }^{3}$ e verificou que apenas $13,7 \%$ da população apresentou um consumo abusivo de álcool, sendo que indivíduos com ensino fundamental incompleto apresentaram índices ainda menores, em torno de $11 \%$. Esses baixos índices podem estar relacionados às metas adotadas pela Organização Mundial de Saúde (OMS), que objetivam reduzir o consumo de álcool, com ações como aumento de preços e restrição da publicidade e propaganda ${ }^{3}$. O percentual de fumantes observado pela PNS também concordou com nosso estu- 
do e foi de apenas $14,5 \%$, sendo revelado um declínio contínuo da prevalência de fumantes no Brasil ${ }^{3}$.

Em relação à nutrição, os indivíduos com dor no ombro apresentaram um perfil regular a positivo, o que indicou um estilo de vida alimentar semelhante aos de indivíduos portadores de DCNT, visto que estudos que avaliaram o estilo de vida de hipertensos e diabéticos mostraram que a maioria desses indivíduos apresenta uma dieta equilibrada ${ }^{23,24}$. O estilo de vida de portadores de dor no ombro também é similar ao dos hipertensos e diabéticos em relação às altas taxas de inatividade física e baixas taxas de consumo de álcool e tabaco ${ }^{23,24}$. Essa similaridade, no entanto, não se deve ao fato de indivíduos com dor no ombro apresentarem DCNT associadas como hipertensão, diabetes, câncer ou doenças respiratórias crônicas, pois a prevalência dessas condições na amostra foi baixa.

De acordo com a $\mathrm{PNS}^{3}, 37 \%$ da população adulta (40 a 59 anos) possui o hábito de consumir carnes com gordura e $38 \%$ possui o hábito de consumir frutas e hortaliças, comportamentos semelhantes aos indivíduos com baixa escolaridade (ensino fundamental incompleto). No presente estudo, $51 \%$ dos indivíduos com dor no ombro, com a mesma faixa etária e escolaridade, relataram consumir cinco porções de frutas e hortaliças diariamente e 56\% relataram evitar alimentos gordurosos. Essa distribuição praticamente equilibrada entre respostas positivas e negativas pode ser preocupante, uma vez que alguns estudos têm relatado associação entre obesidade e maior ocorrência de disfunções musculoesqueléticas, dentre elas a dor no ombro. Em um estudo longitudinal realizado por Nilsen et $\mathrm{al}^{25}$ foi evidenciado que tanto a obesidade como o excesso de peso em homens e mulheres poderiam ser preditores de dor no ombro.

No componente relacionamento social houve uma predominância do perfil positivo quanto a cultivar amigos e se envolver em atividades de lazer em grupo. No entanto, os indivíduos com dor no ombro não se mostraram ativos na sociedade. Em relação ao controle do estresse, que apresentou um perfil igual ou superior à regular, a maioria dos indivíduos demonstrou se alterar em discussões e não equilibrar o tempo de trabalho e lazer. Estes aspectos são relevantes, desde que alguns problemas psicossociais podem ser associados com a dor musculoesquelética ${ }^{7}$. Além disso, o estresse pode conduzir à depressão e ao aumento da ansiedade e alguns resultados da literatura indicam que tais problemas de saúde mental podem reduzir o limiar doloroso e causar maior impacto sobre a capacidade física ${ }^{26}$.

A inatividade física é identificada como a quarta principal causa de morte no mundo e está aumentando em muitos países com grandes implicações para a saúde geral da população ${ }^{27}$. Estilos de vida sedentários apresentam forte associação com a ocorrências das DCNT e são um importante fator de risco relacionado à dor musculoesquelética ${ }^{28}$. No entanto, a relação entre atividade física e dor musculoesquelética é complexa, uma vez que os sintomas podem ser a causa da inatividade, bem como sua consequência ${ }^{26,27}$. Uma possível explicação seria a ocorrência de degeneração articular, devido a menor produção de líquido sinovial para proteger as superfícies articulares, levando à dor, porém não é possível saber se a inatividade desses indivíduos é causa ou consequência da dor no ombro ${ }^{26}$.

Neste estudo, os indivíduos com dor no ombro demonstraram não realizarem com frequência atividades físicas moderadas a intensas, nem realizar duas vezes na semana exercícios de força e alongamento muscular. Ambos os itens se referem ao nível de atividade física recomendado pela OMS para indivíduos de 18 a 64 $\operatorname{anos}^{27}$, o que demonstra uma inatividade física dos indivíduos com dor no ombro para as atividades de lazer, atingindo $68 \%$ de respostas negativas. 
De acordo com a $\mathrm{PNS}^{3}$, a prevalência de inatividade física na população brasileira foi de $46 \%$, sendo observado que a atividade física no lazer está associada a maior escolaridade, sexo masculino e idade jovem. Já a atividade física de deslocamento foi mais frequente entre indivíduos do sexo masculino e com baixa escolaridade ${ }^{3}$, o que pode explicar os resultados do nosso estudo com predomínio de indivíduos com ensino fundamental incompleto, já que houve maior proporção de respostas positivas (65\%) para o item que envolveu atividade física relacionado à transporte.

A inatividade física dos indivíduos com dor no ombro pode ser consequência das dores corporais, reconhecidas como barreiras à prática de atividade física ${ }^{29}$. No entanto, o presente estudo mostrou uma correlação baixa entre a prática de atividade física e a incapacidade do ombro, o que sugere que outros fatores não avaliados podem influenciar o nível de atividade física como o medo de cair, a falta de interesse ou o fato de não gostar da prática de atividade física, bem como o desconhecimento de seus benefícios, atitudes e crenças, inexistência de apoio social, características ambientais e saúde auto percebida ${ }^{30}$.

Assim, considerando que a atividade física regular reduz o risco das DCNTs, é fundamental para o equilíbrio de energia e controle de peso $^{27}$ e proporciona diminuição da dor por meio da liberação de endorfinas, a realização de atividade física deve ser incentivada como forma de prevenir e manter os agravos à saúde, como por exemplo, a condição dolorosa no ombro.

Os profissionais envolvidos com a atenção primária podem tentar identificar precocemente a inatividade física, bem como outros fatores de risco relacionados ao estilo de vida, a fim de orientar seus pacientes na adoção de comportamentos saudáveis. Para tanto, também precisam estar atentos aos fatores modificáveis que podem estar determinado a inatividade física e assim viabilizar a promoção de saúde.

Algumas das limitações desse estudo foram uma possível incompreensão do questionário aplicado, bem como, o fato de basear-se no auto relato dos pacientes, sendo que as práticas de estilo de vida foram descritas como são percebidas pelo indivíduo, com possibilidade de em alguns momentos não ter sido relatado comportamentos menos aceitos socialmente ou tendência a referir hábitos saudáveis, mesmo quando não são adotados. Ainda, o poder do teste pode ser interpretado como baixo. No entanto, o poder do teste pode ser influenciado por quatro variáveis: o nível de significância $\alpha$ adotado, a variabilidade da população, a distância entre o valor real do parâmetro e o considerado verdadeiro na hipótese nula (H0) e o tamanho da amostra. Assim, provavelmente o baixo valor da correlação foi determinante para o valor do poder do teste e não necessariamente o tamanho da amostra, uma vez que a amostra representou $89 \%$ da população de interesse do estudo.

Este estudo tem como potencial ter identificado a necessidade de inclusão de medidas de promoção de saúde e prevenção de fatores de risco relacionados ao estilo de vida, principalmente, quanto ao incentivo da prática de atividade física, seguida de orientações em relação à alimentação e ao manejo do estresse, como complementar ao tratamento das disfunções do complexo do ombro no serviço público de fisioterapia de nível secundário.

O estudo concluiu que indivíduos com dor no ombro atendidos em um serviço de fisioterapia de nível secundário apresentam um perfil de estilo de vida de ruim a regular, sendo que uma proporção considerável de indivíduos apresentou um estilo de vida desfavorável principalmente para a prática de atividade física, bem como para controle do estresse e nutrição. Além disso, o estilo de vida referente à atividade física mostrou correlação baixa com a incapacidade do ombro nesses pacientes. 


\section{Contribuição dos autores}

J. Paglioto: elaboração da pesquisa, levantamento da literatura, coleta dos dados, análise e interpretação dos dados, redação do artigo, aprovação da versão final do artigo submetido. J. Martins: elaboração da pesquisa, levantamento da literatura, análise e interpretação dos dados, contribuição intelectual importante na revisão crítica do artigo, aprovação da versão final do artigo submetido. C. S. Souza: elaboração da pesquisa, coleta dos dados, análise e interpretação dos dados, redação do artigo, aprovação da versão final do artigo submetido. A. S. Oliveira: elaboração da pesquisa, análise e interpretação dos dados, contribuição intelectual importante na revisão crítica do artigo e aprovação da versão final do artigo submetido.

\section{Referências}

1. Põlluste K, Aart A, Kallikorm R, Kull M, Kärberg K, Müller R, Ots-rosenberg M, Tolk A, Uhlinova J, LemberM. Adverse lifestyle and health-related quality of life: gender differences in patients with and without chronic conditions. Scand J Public Health. 2016; 44: 209-16.

2. World Health Organization. Envelhecimento ativo: uma política de saúde / World Health Organization; tradução Suzana Gontijo. - Brasília: Organização Pan-Americana da Saúde, 2005. 60p.

3. Malta DC, Andrade SSCA, Stopa SR, Pereira CA, Szwarcwald CL, Júnior JBS, ET AL. Estilos de vida da população brasileira: resultados da Pesquisa Nacional de Saúde. Epidemiol Serv Saúde. 2015; 24(2):217-26.

4. Organização Mundial da Saúde. Organização Pan-Americana da Saúde. Prevenção de doenças crônicas: um investimento vital. Genebra: Organização Mundial da Saúde; 2005. 36 p.

5. Malta DC Neto OLM, Júnior JBS. Apresentação do plano de ações estratégicas para o enfrentamento das doenças crônicas não transmissíveis no Brasil, 2011 a 2022. Epidemiol Serv Saúde. 2011; 20(4):425-38.

6. March L, Smith EU, Hoy DG, Cross MJ, Sanchez-Riera L, Blyth F, Buchbinder R, Vos T, Woolf AD. Burden of disability due to musculoskeletal (MSK) disorders. Best Pract Res Clin Rheumatol. 2014;28:353-66.

7. Puroila A, Lifestyle-Factors in Adolescence as Predictors of Number of Musculoskeletal Pain Sites in Adulthood: A 17-Year Follow-Up Study of a Birth Cohort Pain Med. 2015;16:1177-85.

8. Shiri R, Karppinen J, Leino-Arjas P. The association between obesity and low back pain: a meta-analysis. Am J Epidemiol. 2010;171:135-54.

9. Haukka E, Ojajarvi A, Takala E, Viikari-Juntura E, Leino-Arjas P. Physical workload, leisuretime physical activity, obesity and smoking as predictors of multisite musculoskeletal pain. A 2-year prospective study of kitchen workers. Occup Environ Med. 2012; 69:485-92.

10. McBeth J, Jones K. Epidemiology of chronic musculoskeletal pain. Best Pract Res Clin Rheumatol. 2007;21(3):403-25.

11. Karel YHJM, Scholten-Peeters WGM, Thoomes-de Graaf M, Duijn E, Ottenheijm RPG, Van den Borne MPJ, et al. Current management and prognostic factor in physioteraphy practice for patients with shoulder pain: design of a prospective cohort study. BMC Musculoskelet Disord. 2013; 14(62):1-7.

12. Marcondes FB, Rosa SG, Vasconcelos RA, Basta A, Freitas DG, Fukuda TY. Força do manguito rotador em indivíduos com síndrome do impacto comparado ao lado assintomático. Acta Ortop Bras. 2011; 19(6): 333-7.

13. Brox JI. Shoulder pain. Best Pract Res Clin Rheumatol 2003;17(1):33-56.

14. Hill CL, Gill TK, Shanahan EM, Taylor AW. Prevalence and correlates of shoulder pain and stiffness in a population-based study: the North West Adelaide Health Study. Int J Rheum Dis. 2010;13:215-22.

15. Gill TK, Shanahan EM, Taylor AW, Buchbinder R, Hill CL. Shoulder Pain in the Community: An Examination of Associative Factors Using a Longitudinal Cohort Study. Arthritis Care Res. 2013; 65(12):2000-7.

16. Júnior J. Fisioterapia e saúde coletiva: desafios e novas responsabilidades profissionais. Ciênc Saúde Coletiva. 2010;15(1):1627-36. 
17. Martins J, Napoles BV, Hoffman CB, Oliveira AS. Versão Brasileira do Shoulder and Disability Índex: tradução, adaptação cultural e confiabilidade. Rev Bras Fisioter. 2010;14(6):527-36.

18. Puga VO, Lopes AD, Shiwa SR, Alouche SR, Costa LO. Clinimetric testing supports the use of 5 questionnaires adapted into Brazilian Portuguese for patients with shoulder disorders. J Orthop Sports Phys Ther. 2013;43(6):404-13.

19. Nahas MV, Barros MVG, Francalacci V. O Pentáculo do Bem Estar - Base Conceitual para Avaliação do Estilo de Vida de Indivíduos ou Grupos. Rev Bras Ativ Fís Saúde. 2000;5(2):48-59.

20. Both J, Borgatto AF, Nascimento JV, Sonoo CN, Lemos CAF, Nahas MV. Validação da Escala "Perfil do Estilo de Vida Individual". Rev Bras Ativ Fís Saúde. 2008;13(1):5-14.

21. Silveira PM, Borgatto AF, Andrade DF, Both J, Nascimento JV. Escala de avaliação do perfil do estilo de vida por meio da teoria da resposta ao item. Rev Educ Fís/UEM; 2015. 26(4):519-27.

22. Domholdt E. Physical Therapy Research: Principles and Applications. 2nd ed. Philadelphia: Saunders, 2000.

23. Teixeira ER, Silva JC, Lamas AR, Matos RM. O estilo de vida do cliente com hipertensão arterial e o cuidado com a saúde. Esc Anna Nery R Enferm. 2006; 10(3):378-84.

24. Cotta RMM, Batista KCS, Reis RS, Souza GA, Dias G, Castro FAF, et al. Perfil sociossanitário e estilo de vida de hipertensos e/ou diabéticos, usuários do Programa de Saúde da Famíliano município de Teixeiras, MG. Ciênc Saúde Coletiva. 2009; 14(4):125160.

25. Nilsen TI, Holtermann A, Mork PJ. Physical exercise, body mass index, and risk of chronic pain in the low back and neck/shoulders: longitudinal data from the Nord-Trondelag Health Study. Am J Epidemiol. 2011;174(3):267-73.

26. Dean E, Soderlund, A. What is the role of lifestyle behaviour change associated with noncommunicable disease risk in managing musculoskeletal health conditions with special reference to chronic pain? BMC Musculoskelet Disord. 2015; 16(87):1-7

27. World Health Organization. Global recommendations on physical activity for health. Geneva; 2010.

28. De Vitta A, Neri A, L, Padovani CR. Nível de atividade física e desconfortos musculoesqueléticos percebidos em homens e mulheres, adultos e idosos. Rev Bras Fisioter. 2003;17(1):45-52.

29. Lopes MA, Krug RR. Bonetti A, Mazo GZ. Barreiras que influenciaram a não adoção de atividade física por longevas. Rev Bras Ciênc Esporte. 2016;38(1):76-83.

30. Krug RR, Lopes MA, Mazo GZ, Marchesan M. Pain impairs the practice of regular physical activities in the perception of longevous women. Rev Dor. São Paulo, 2013;14(3):192-95.

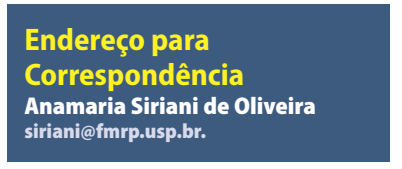

Endereço para

espondência

Anamaria Siriani
siriani@fmrp.usp.br.

de Ribeirão Preto da Universidade de São

Paulo, Avenida dos Bandeirantes, 3900,

Monte Alegre, CEP:14049-900, Ribeirão

Preto/SP - Brasil.

Telefone: 16 3315-4415; Fax: 16 3315-4413.

RECEBIDO 04/10/2016

REVISADO $16 / 12 / 2016$

$21 / 12 / 2016$

APROVADO $21 / 12 / 2016$ 